\title{
Validade da Estrutura Fatorial de Uma Bateria de Avaliação de Altas Habilidades
}

\author{
Walquiria de Jesus Ribeiro \\ Tatiana de Cássia Nakano \\ Pontificia Universidade Católica de Campinas \\ Campinas, SP, Brasil \\ Ricardo Primi \\ Universidade São Francisco \\ São Paulo, SP, Brasil
}

\begin{abstract}
RESUMO
O tema das altas habilidades/superdotação, no cenário da avaliação psicológica, tem se constituído um desafio, haja vista a sua multidimensionalidade. Nesse estudo buscou-se investigar a validade de construto da Bateria para Avaliação das Altas Habilidades. Participaram desta pesquisa 588 alunos do $2^{\circ}$ ano do ensino fundamental ao $3^{\circ}$ ano do ensino médio, ambos os sexos, divididos em grupo controle $(\mathrm{N}=470)$ e grupo critério $(\mathrm{N}=118)$, com média de idade de 11,11 anos. O instrumento foi composto por quatro subtestes de inteligência (Raciocínio Verbal, Abstrato, Numérico e Lógico) e dois subtestes de criatividade (Completando Figuras e Criação de Metáforas). Obteve-se através da análise fatorial exploratória, três fatores explicando $70,72 \%$ da variância total, representando uma medida de criatividade verbal, uma medida de inteligência e uma última, de criatividade figural. No geral, foram observadas correlações baixas entre os fatores. Conclui-se que o instrumento apresentou validade de construto para avaliação dos construtos envolvidos no fenômeno.
\end{abstract}

Palavras-chave: Superdotados. Inteligência. Criatividade. Avaliação psicológica. Testes psicológicos.

\section{ABSTRACT}

\section{Validity of the Factor Structure of a Battery of Assessment of High Abilities}

The theme of high abilities/giftedness in psychological assessment context, has been a challenge, given its multidimensionality. This study we sought to investigate the construct validity of the Battery for Assessment of High Ability. The sample was composed by 588 students of the $2^{\text {nd }}$ year of elementary school to $3^{\text {rd }}$ year of high school, both sexes. They were divided into a control group $(\mathrm{N}=470)$ and test group $(\mathrm{N}=118)$, with a mean age of 11.11 years. The instrument was composed of four subtests of intelligence (Verbal, Abstract, Numerical and Logical Reasoning) and two subtests of creativity (Construction of Figures and Metaphors Creation). The results of exploratory factor analysis indicated three factors explaining $70.72 \%$ of the total variance, representing a measure of verbal creativity, a measure of intelligence and a measure of figural creativity. In general, low correlations were observed between the factors. We conclude that the instrument presented construct validity for assessing three constructs involved in this phenomenon.

Keywords: Gifted. Intelligence. Creativity. Psychological assessment. Psychological tests.

\section{RESUMEN}

\section{Validez de la Estructura Factorial de Una Batería de Evaliación de Altas Capacidades}

El tema de altas capacidades/superdotación, en el escenario de la evaluación psicológica, ha sido un desafío, dada su multidimensionalidad. En este estudio hemos tratado de investigar la validez de constructo de la Batería de Evaluación de Alta Capacidad. La muestra fue de 588 estudiantes de la 2 da. año de la escuela primaria a tercero. año de la escuela secundaria, ambos sexos fueron divididos en un grupo control $(\mathrm{N}=470)$ y el grupo de prueba $(\mathrm{N}=118)$, con una edad media de 11,11 años. El instrumento consta de cuatro pruebas de inteligencia (Razonamiento Verbal, Abstracto, numérico y lógico) y dos subpruebas de la creatividad (Figuras redondeo y Metáforas creación). Se obtuvo mediante un análisis factorial exploratorio, tres factores que explican $70,72 \%$ de la varianza total, lo que representa una medida de la creatividad verbal, una medida de la inteligencia y la creatividad final, figural. En general, se observaron bajas correlaciones entre los factores. Llegamos a la conclusión de que el instrumento tenía validez aparente para evaluar constructos implicados en este fenómeno.

Palabras clave: Talento. Inteligencia. Creatividad. Evaluación psicológica. Pruebas psicológicas. 
A investigação científica sobre superdotação foi impulsionada a partir das pesquisas desenvolvidas no século XIX por Galton e Binet, cujos interesses pelos processos mentais os conduziram a estudos relevantes sobre o intelecto humano e à construção dos primeiros testes para avaliação da inteligência (Almeida e Capellini, 2005). Em 1985, reformulações na teoria de Binet, empreendidas por Lewis Terman, trouxeram estimativas de escores de QI (Quociente Intelectual) acima de 135 como indicativo na avaliação de crianças superdotadas, associando nessa concepção, superdotação à inteligência superior. No entanto, ainda que em alguns estudos esta definição tenha se mantido presente, caracterizando o desempenho intelectual como parâmetro de identificação padrão da superdotação, especialistas têm apontado limitações nesse modelo de avaliação, sobre o qual vários teóricos destacam a importância de serem avaliadas também outras habilidades cognitivas (Gardner, 2001; Roazzi et al., 2007; Sternberg, 1984, 2005). Embora, tradicionalmente, a habilidade intelectual sempre tenha sido enfatizada nesse contexto, autores apontam ainda, a necessidade de ampliar o diagnóstico de modo a incluir outros processos além da inteligência, concebendo a superdotação em sua natureza multidimensional (Holling e Kuhn, 2008; Pfeiffer e Blei, 2008; Li et al, 2009).

No cenário dos modelos mais atuais, a Teoria dos Três Anéis (Renzulli, 1978) caracteriza a superdotação a partir da interação entre três componentes: habilidade intelectual acima da média, envolvimento com a tarefa e criatividade, identificando ainda, duas categorias de superdotação: acadêmica e produtivo-criativa, associando a primeira ao bom desempenho acadêmico e elevado repertório de conteúdo, e a segunda, ao pensamento integrado, indutivo e desenvolvimento de produtos originais (Renzulli, 2004). Considerando a sua valorização no meio científico, tal modelo foi utilizado como fundamento nas políticas públicas brasileiras, permeando o conceito adotado pelo Ministério da Educação, para o qual, alunos com altas habilidades são aqueles que apresentam um alto potencial, combinado ou isolado, nas áreas intelectual, acadêmica, de liderança e psicomotricidade, além de manifestar uma elevada criatividade, um alto envolvimento com a aprendizagem e também com a realização de tarefas de seu interesse (MEC, 2010).

Com vista à contemplação dessas características, a literatura tem recomendado a realização de um processo de avaliação multifatorial que considere informações provenientes de distintas medidas psicométricas, complementadas por observações gerais, avaliação de portfólios e de trabalhos produzidos, além do julgamento de professores, cujas informações podem auxiliar na decisão acerca da presença de critérios indicativos de superdotação (Gridley e Treloar, 1984; Pfeiffer e Blei, 2008). Entretanto, essa não é a situação comumente encontrada, uma vez que, conforme destacado por Bracken e Brown (2006), na falta de um consenso acerca das definições do fenômeno e na ausência de medidas adequadas de avaliação dos alunos superdotados, a dificuldade na identificação tem se mantido.

Diante da importância reconhecida do fenômeno, um aspecto que chama a atenção é a inexistência, até o momento, de instrumentos específicos aprovados pelo Conselho Federal de Psicologia para sua avaliação. Por esse motivo, as pesquisas na área têm, em sua maior parte, feito uso de instrumentos não específicos para essa finalidade, ainda que uma avaliação mais específica se mostre necessária. Essa lacuna deve ser sanada pelos profissionais que trabalham na área da avaliação psicológica, visto que psicólogos têm sido desafiados a fazer predições sobre o potencial desses estudantes ou sua futura performance. Embora tradicionalmente realizada em outros países, de forma que importantes avanços têm sido obtidos tanto em relação à conceituação quanto à avaliação desse fenômeno, o quadro atual mostra que nosso país encontra-se defasado em relação à temática.

Vale ressaltar que, embora diversas leis federais venham propondo, há vários anos, a importância de ações de intervenção voltadas ao atendimento de crianças com altas habilidades, a dificuldade em determinar quem seriam estas crianças e, principalmente de criar formas para avaliá-las apropriadamente, tem atuado de forma a impedir que muitas delas tenham acesso a programas de estimulação e desenvolvimento, devendo ser este um dos desafios atuais da Psicologia. Ressaltese o fato de que a carência desse tipo de instrumental pode resultar numa dificuldade para identificar e encaminhar as crianças com altas habilidades para atendimento especializado de modo a ter seu potencial melhor aproveitado. Essa lacuna faz com que estudos e pesquisas na área sejam de imenso valor frente ao benefício que podem trazer para esses potenciais que vem sendo desperdiçados no país, sendo objetivo da presente pesquisa o desenvolvimento de estudos de busca por evidências de validade de uma bateria para avaliação da superdotação em crianças e adolescentes com idades entre 10 e 15 anos.

\section{MÉTODO}

\section{Participantes}

Utilizou-se uma amostra de conveniência, composta por 588 alunos do segundo ano do ensino 
fundamental ao terceiro ano do ensino médio, divididos em grupo controle, formado por 470 alunos de sala de ensino regular de escolas das cidades de São Luis (MA), Campinas (SP) e Cordeirópolis (SP) e grupo critério, constituído por 118 alunos, participantes de sete Salas de Recurso do Programa de Atendimento ao Aluno com Altas Habilidades da Secretaria de Estado de Educação do Distrito Federal, identificados previamente pelos métodos de avaliação do respectivo Programa. Ressalta-se que neste grupo, participaram alunos com Habilidades na área Acadêmica e na área de Talentos Artísticos. Do total, 52,5\% $(\mathrm{N}=309)$ eram do sexo feminino e $47,5 \%(\mathrm{~N}=279)$ do sexo masculino, com idade média de 11,11 anos $(\mathrm{DP}=2,02)$, com mínima de 7 e máxima de 17 anos.

\section{Material}

A Bateria para Avaliação das Altas Habilidades (Nakano e Primi, 2012) é composta por quatro subtestes de inteligência (Raciocínio Verbal, Abstrato, Numérico e Lógico) e dois subtestes de criatividade (Teste Completando Figura e Teste de Criação de Metáforas).

Raciocínio Verbal ( $R V)$ : doze questões, cada uma contendo frases com pares de palavras relacionadas entre si, estando um dos pares, incompleto. Por analogia ao primeiro par, deverá ser escolhida, dentre cinco alternativas, a palavra que completará o segundo par. Tempo de execução aproximado de 12 minutos. Atribui-se um ponto para cada resposta correta.

Raciocínio Abstrato (RA): doze questões, cada uma contendo conjuntos formados por dois pares de figuras, estando um deles, incompleto. Por analogia ao primeiro par, deverá ser assinalada, dentre cinco opções, a figura correta para completar o segundo par. Tempo de execução aproximado de 12 minutos. Atribui-se um ponto para cada resposta correta.

Raciocínio Numérico $(R N)$ : doze sequências numéricas, faltando os dois últimos números de cada uma delas. Por identificação da relação aritmética existente entre os números, deverão ser descobertos aqueles que completarão a série. As respostas são anotadas no caderno de resposta. Tempo de execução aproximado de 12 minutos. Atribui-se um ponto para cada resposta correta.

Raciocínio Lógico (RL): doze questões apresentando problemas de ordem prática e cotidiana, para os quais deverá ser encontrada uma solução. $\mathrm{O}$ número de respostas varia a cada questão e as mesmas deverão ser escritas no caderno de respostas. Tempo de execução aproximado de 12 minutos. A pontuação para cada questão será dada em função do total de respostas respondidas corretamente.
Teste Completando Figura: avalia a criatividade figural através da realização de desenhos fazendo-se uso de um estímulo incompleto, o qual deverá ser completado à vontade do examinando. Dez estímulos são fornecidos na atividade, para a qual o tempo limite de execução é de 10 minutos. A avaliação de cada desenho é feita, considerando-se a ocorrência de onze características/indicadores criativos: Fluência (quantidade de desenhos relevantes), Flexibilidade (diversidade de categorias), Elaboração (quantidade de detalhes que compõe o desenho), Originalidade (quantidade de ideias incomuns), Expressão de Emoção (sentimentos apresentados nos desenhos ou nos títulos), Fantasia (expressão de seres imaginários, personagens de contos de fada, etc.), Movimento (expressão explícita de movimento), Perspectiva Incomum (realização de desenhos sob ângulos diferentes), Perspectiva Interna (detalhes que mostrem visão interna dos desenhos), Uso de Contexto (desenho inserido num contexto) e Títulos Expressivos (títulos que refletem abstração na nomeação dos desenhos). As pontuações em tais características são posteriormente agrupadas em três fatores: (1) Elaboração - Fantasia, Perspectiva Incomum, Perspectiva Interna, Uso de Contexto e Elaboração); (2) Emocional - Expressão de Emoção, Movimento e Títulos Expressivos e (3) Cognitivo - Fluência, Flexibilidade e Originalidade.

Teste Criação de Metáforas: avalia a criatividade verbal através de cinco frases incompletas as quais deverão ser completadas com uma palavra, de maneira a formar uma metáfora. Para cada frase, poderão ser fornecidas até quatro respostas, devendo ser explicitada a relação metafórica estabelecida. Dois indicadores são considerados na correção do teste: Qualidade, composta pela avaliação da equivalência (efetividade na associação de idéias elaboradas) e remoticidade (distância entre os campos semânticos, que tornam a metáfora mais surpreendente), cuja pontuação varia entre 1 a 3, e Flexibilidade, relacionada à utilização de diferentes categorias de resposta, cuja pontuação varia entre 1 e 4 . A correção é feita por juízes que atribuem as pontuações de qualidade e flexibilidade a cada idéia. Os escores são calculados por meio de um modelo de Teoria de Resposta ao Item (Rasch-Linacre Many-Facet Measurement) que contém parâmetros para a dificuldade dos itens, a severidade dos juízes e a habilidade do sujeito (Primi, Miguel, Couto e Muniz, 2007). Tempo de execução médio de 15 minutos.

\section{Procedimentos}

Inicialmente a pesquisa teve sua execução aprovada pelo Comitê de Ética em Pesquisa, sob 
número 0630/11. Após autorização das escolas e permissão dos pais, através da assinatura do Termo de Consentimento Livre e Esclarecido, o instrumento foi aplicado coletivamente, na amostra controle, em grupos de alunos de mesma escolaridade e, na amostra critério, em grupos de alunos presentes nas Salas de Recursos no dia da aplicação. As Salas de Recurso contempladas localizam-se no Plano Piloto e Cidades Satélites do Distrito Federal, dentre as quais, duas atendem alunos na área de habilidades acadêmicas, duas na área de talentos artísticos e três, atendem as duas áreas, cada uma, acompanhada por professores específicos.

O instrumento foi aplicado em uma única sessão com duração aproximada de $1 \mathrm{~h} 40 \mathrm{~min}$, obedecendo-se o tempo limite indicado para cada subteste. Após as informações gerais e esclarecimento de dúvidas, apresentou-se o primeiro subteste com as respectivas instruções. Ao esgotar-se o tempo do subteste, os alunos eram instruídos a interromper a tarefas e passar, após as instruções, para o subteste seguinte, seguindo-se dessa forma, até a finalização de todos os subtestes. A sequência de aplicação teve inicio com a Prova de Raciocínio Verbal, seguida pela Prova de Raciocínio Abstrato, Teste Completando Figura, Teste de Criação de Metáforas, Prova de Raciocínio Numérico e Prova de Raciocínio Lógico.

Paralelamente à aplicação dos instrumentos, procedeu-se à correção dos subtestes, utilizando o sistema de pontuação baseado nos parâmetros anteriormente descritos para cada um deles. Para a correção dos subtestes de raciocínio utilizou-se o sistema de pontuação considerando o número total de acertos em cada um dos quatro subtestes e para o subteste de criatividade figural, foram pontuadas a ocorrência das características em cada uma dos desenhos produzidos pelos participantes e posteriormente somadas, a fim de se obter um quantitativo total em cada característica. Por fim, o Teste Criação de Metáforas, obedeceu a um sistema de correção que contou com a participação de cinco juízes, os quais, através de meio eletrônico, receberam planilhas com todas as respostas dos alunos, sendo as mesmas avaliadas atribuindo-se uma nota (0 a 3) para o fator Qualidade e outra nota (1 a 4) para o fator Flexibilidade. Ao final, estas avaliações passaram por tratamento estatístico e estimação de uma nota de um juiz "médio", por meio do uso da Teoria de Resposta ao Item.

\section{RESULTADOS}

Através da análise fatorial exploratória buscou-se evidência de validade da estrutura interna da Bateria para Avaliação das Altas Habilidades partindo-se de três hipóteses iniciais. A primeira considerando a existência de três fatores diferentes - inteligência, criatividade figural e criatividade verbal - a segunda, apenas dois fatores - inteligência e criatividade. E, por último a existência de um fator geral.

Para a realização das análises foram tomadas as medidas das Provas de Raciocínio (RA-Abstrato; RV-Verbal; RN-Numérico e RL-Lógico), do Teste Criação de Metáfora (QLD_MET - Qualidade e FLX_MET - Flexibilidade) e do Teste Completando Figura, considerado o modelo fatorial de correção baseado em três fatores (ELB_FIG - Elaboração, EMO_FIG - Emocional e COG_FIG - Cognitivo).

A análise fatorial exploratória foi feita no Mplus (Muthén e Muthén, 2010), empregando-se o método de estimação por máxima verossimilhança e o procedimento de rotação oblíqua Geomin. Uma vantagem do Mplus é a obtenção de índices de ajuste usados em analises fatoriais confirmatórias, permitindo-se avaliar o ajuste do modelo. Foram examinados os índices $\chi^{2}$ (qui-quadrado), o Comparative Fit Index (CFI), Root-Mean-Square Error of Approximation (RMSEA) e o Standardized Root Mean Square Residual (SRMR). Efetuou-se também uma análise paralela com a replicação de 50 matrizes de correlação, geradas aleatoriamente, como recurso para avaliar o número de fatores a serem extraídos.

O resultado da análise paralela é apresentado na Figura 1. Os índices de ajuste para os três modelos testados foram: (a) fator $\mathrm{g}$ : $\chi^{2}=844,3$; $\mathrm{CFI}=0,63$; RMSEA $=0,22$ e $\mathrm{SRMR}=0,10$; (b) dois fatores: $\chi^{2}=172,8 ; \mathrm{CFI}=0,93$; $\mathrm{RMSEA}=0,12$ e $\mathrm{SRMR}=0,07$; (c) três fatores $\chi^{2}=15,2$; CFI $=0,99$; RMSEA $=0,02$ e $\mathrm{SRMR}=0,01$. Como se pode perceber, o modelo de três fatores apresentou os melhores índices de ajuste, sendo que o modelo de um fator apresentou índices inaceitáveis, de modo a sugerir a existência de um modelo multidimensional.

Foram analisadas as estruturas de dois e três fatores, chegando-se à conclusão de que a estrutura de três fatores é a mais coerente do ponto de vista teórico e do critério estatístico. Os três fatores resultaram em eigenvalues de 3,737; 1,535 e 1,093 a partir da rotação oblíqua, conforme mostra a Figura 1, explicando variância total de 70,72\% (41,52; 17,05 e 12,14).

Procedendo-se às analises, encontra-se disposta na Tabela 1, a estrutura fatorial do instrumento em estudo, para a qual estão demonstradas as cargas dos componentes de cada fator, tendo-se considerado para cada um deles as cargas fatoriais significativas (obtida a partir da rotação Geomin). 


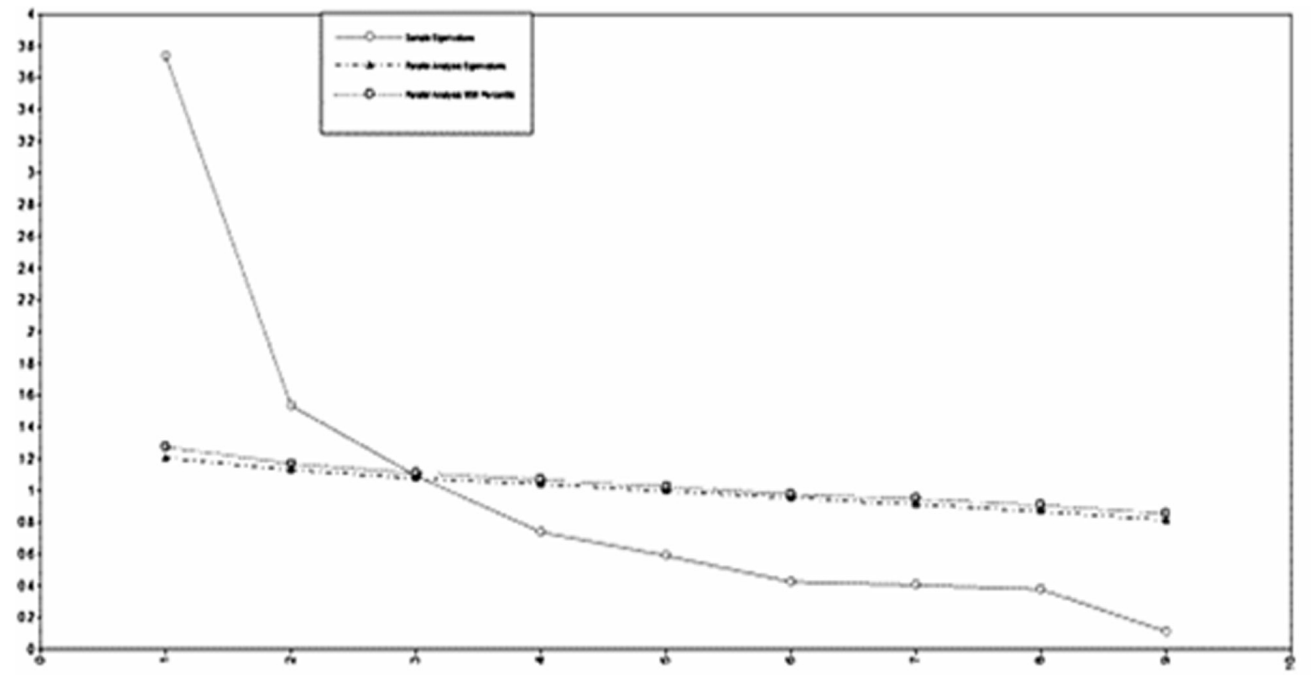

Figura 1. Gráfico de sedimentação com os três fatores

TABELA 1

Estrutura fatorial da Bateria de Avaliação das Altas Habilidades

\begin{tabular}{lccc}
\hline & \multicolumn{3}{c}{ Fatores } \\
\cline { 2 - 4 } \multicolumn{1}{c}{ Variáveis } & $\begin{array}{c}1 \\
\text { criatividade } \\
\text { verbal }\end{array}$ & $\begin{array}{c}2 \\
\text { inteligencia }\end{array}$ & $\begin{array}{c}3 \\
\text { criatividade } \\
\text { figural }\end{array}$ \\
\hline FLX_MET & $0,903^{*}$ & \\
QLD_MET & $0,457^{*}$ & $0,367^{*}$ & \\
ELB_FIG & & $0,248^{*}$ & $0,433^{*}$ \\
EMO_FIG & & $0,097^{*}$ & $0,459^{*}$ \\
COG_FIG & & $-0,016^{*}$ & $0,777^{*}$ \\
RA & & $0,759^{*}$ & \\
RV & & $0,788^{*}$ & \\
RN & & $0,780^{*}$ & \\
RL & & $0,739^{*}$ & \\
\hline
\end{tabular}

* Nível de significância: 0,05 .

Percebe-se desse modo, que o Fator 1 se constitui pelas medidas de FLX_MET (Flexibilidade) e QLD_MET (Qualidade Metafórica), ambas avaliadas no Teste de Criação de Metáforas, caracterizando, portanto, um fator associado à criatividade verbal, relacionado à habilidade de manipulação no trato com conteúdo verbal e categorias semânticas envolvidas em associações, aproximações e transferências de significados. As medidas RA (Raciocínio Abstrato), RV (Raciocínio Verbal), RN (Raciocínio Numérico) e RL (Raciocínio Lógico), todas avaliadas nos subtestes de raciocínio, compõem o Fator 2, caracterizando um construto associado à inteligência, relacionado à capacidade de uso de recursos cognitivos, inteligência fluída e cristalizada. Por fim, o Fator 3 apresenta-se composto pelas medidas do Teste Completando Figura:
ELB_FIG (Elaboração), EMO_FIG (Emocional) e FGCOG_FIG (Cognitivo), medidas estas que contemplam o modelo de avaliação da criatividade figural empregado neste estudo. O Fator 3 mostrou-se associado ao construto criatividade figural.

Quanto à análise acerca da correlação entre os três fatores encontrados, os resultados apontam um valor de 0,346 entre criatividade verbal e inteligência; 0,131 (com nível de significância de 0,05) entre inteligência e criatividade figural e de 0,039 entre criatividade verbal e criatividade figural. Tais resultados permitem verificar a existência de aspectos comuns entre os três construtos (variância comum), mais notadamente entre criatividade verbal e inteligência, assim como particularidades entre os mesmos. Esta mesma análise foi conduzida visando-se identificar as correlações entre as medidas dos subtestes de criatividade verbal, figural e inteligência, cujos resultados encontram-se dispostos na Tabela 2.

Analisando-se de forma mais detalhada as correlações entre as várias medidas, pode-se observar, em primeiro lugar, que as maiores correlações são encontradas entre as medidas de cada subteste, destacando-se, por exemplo, o valor de 0,878 entre as duas medidas do teste de metáforas. A situação se repete em relação aos três fatores do teste figurativo, cujos valores oscilam entre 0,251 e 0,363 e entre os subtestes de raciocínio (entre 0,579 e 0,603).

Quanto às correlações entre os construtos, as correlações entre inteligência e criatividade apresentaram valores de magnitude mais alta quando estabelecidas com a criatividade verbal. Correlações com magnitude moderada foram encontradas entre as duas medidas do Teste Criação de Metáforas e quatro 
TABELA 2

Correlação entre as medidas dos subtestes de criatividade verbal, criatividade figural e inteligência

\begin{tabular}{lccccccccc}
\hline & $F L X \_M E T$ & QLD_MET & ELB_FIG & EMO_FIG & COG_FIG & $R A$ & $R V$ & $R N$ & $R L$ \\
\hline FLX_MET & - & & & & & & & & \\
QLD_MET & 0,878 & - & & & & & & & \\
ELB_FIG & 0,130 & 0,153 & - & & & & & & \\
EMO_FIG & 0,102 & 0,096 & 0,251 & - & & & & \\
COG_FIG & 0,046 & 0,043 & 0,357 & 0,363 & - & & & \\
RA & 0,391 & 0,373 & 0,248 & 0,123 & 0,051 & - & & \\
RV & 0,422 & 0,444 & 0,216 & 0,118 & 0,053 & 0,603 & - & \\
RN & 0,352 & 0,376 & 0,250 & 0,111 & 0,060 & 0,600 & 0,579 & - & \\
RL & 0,498 & 0,440 & 0,213 & 0,145 & 0,113 & 0,574 & 0,603 & 0,603 & - \\
\hline
\end{tabular}

medidas dos subtestes de raciocino (Verbal, Abstrato, Numérico e Lógico), notadamente RV e RL. Situação diferente é encontrada em relação à inteligência e criatividade figural, visto que os valores de magnitude mostraram-se mais baixos. Interessantemente o fator Elaboração foi o que apresentou maiores correlações com os quatro subtestes de raciocínio, sendo o fator Cognitivo o que menos associou-se às medidas de inteligência, contrariamente ao esperado. Curiosamente, os valores mais baixos foram encontrados em relação às medidas pertencentes aos dois tipos de criatividade.

\section{DISCUSSÃO}

A partir das extrações realizadas nesta análise, verificou-se que a Bateria para Avaliação das Altas Habilidades contempla, através dos seis subtestes, uma estrutura multidimensional, que engloba a avaliação de três construtos diferentes - criatividade verbal, inteligência e criatividade figural, representados respectivamente, pelos Fatores 1, 2 e 3, ainda que valores baixos ou moderados de correlação tenham sido encontrados entre eles. A partir de tais resultados, a discussão acerca de três constatações se mostram importantes: (1) a separação entre os construtos inteligência e criatividade, (2) a baixa correlação entre as medidas de criatividade e (3) a relação entre criatividade verbal e inteligência.

Em relação ao primeiro ponto, os resultados encontrados apontam mais para a independência entre os construtos do que para sua proximidade, dadas as baixas correlações encontradas e pela separação das medidas, em diferentes fatores, na análise fatorial. Tal resultado encontra amparo, parcialmente, em uma vertente de pesquisadores que defendem a idéia de que criatividade e inteligência seriam construtos diferentes, independentes e separados conceitualmente (Colom, 2008; Getzels e Jackson, 1962; Hattie e Rogers,
1986; Sternberg, 1984), quando se considera a baixa correlação encontrada entre os subtestes de raciocínio e criatividade figural, sendo que, no entanto, valores moderados foram encontrados em relação à criatividade verbal, a qual mostra-se mais próxima do construto inteligência. Pesquisadores dessa corrente defendem que o QI por si só não poderia ser visto como único fator para a determinação do talento criativo (Sternberg e O'Hara, 2000), uma vez que a criatividade pode ser vista suficientemente distinta da inteligência, podendo, inclusive, servir como preditor concorrente para a realização escolar (Rindermann e Neubauer, 2004).

A revisão da literatura também aponta diversos estudos nos quais a correlação entre os conceitos aponta para relações não significativas ou valores baixos (Aguirre e Conners, 2010; Gonçalves e Fleith, 2011; Nakano e Brito, no prelo; Wechsler, Nunes, Schelini, Ferreira e Pereira, 2010). Neste sentido, a explicação das diferenças entre os construtos, como afirmam Sternberg e O'Hara (2000) poderia se situar no fato de que "a inteligência tem sido concebida como crucial na adaptação a ambientes existentes, enquanto que a criatividade, a qual envolve a produção de idéias ou produtos novos e úteis, tem sido vista como crucial na modificação e modelagem do ambiente" (p. 611). Assim, Wechsler, Nakano, Nunes e Minervino (2010), após revisão das pesquisas encontradas na literatura, mostraram a necessidade da avaliação tanto da inteligência quanto da criatividade, dado o fato de ambas habilidades constituírem-se em aspectos essenciais, porém distintos, do funcionamento cognitivo.

Entretanto, ainda que tal posicionamento seja defendido por diversos autores na literatura científica nacional e internacional, cautela deve ser recomendada na interpretação dos dados, visto que, ainda que a relação entre inteligência e criatividade, venha sendo amplamente debatida e estudada (Albert e 
Runco, 1999), ainda é um tema que tem se mostrado controverso no domínio da Psicologia e dos processos cognitivos (Pereira, 2001). Segundo Kim (2005), o questionamento aos resultados encontrados na literatura ampara-se na constatação de que o valor e a significância dessa relação irão depender do tipo de criatividade e de inteligência que são medidas, de forma que diferentes resultados são relatados de acordo com o tipo de estudo, sendo que, os achados, aparentemente contraditórios, podem ser explicados, em parte, pela heterogeneidade das medidas empregadas e tipo de população estudada. Isso porque três diferentes vertentes têm sido encontradas: autores que uma afirmam a existência de uma alta relação entre criatividade e inteligência, outros que defendem a independência entre esses dois construtos e ainda autores que afirmam a existência de relação somente a partir de um certo nível dos dois construtos (Hattie e Rogers, 1986), Desse modo, o consenso mostra-se longe de ser atingido, de forma que as investigações atuais seguem questionando a existência dessa relação e o nível da associação, além da sua estabilidade no tempo e possibilidade de generalização para populações distintas (Elisondo e Donolo, 2010).

A revisão das teorias e dos resultados que vêm sendo apontados na literatura, internacional e nacional, mostrou que, dependendo de como os construtos são medidos, do instrumento utilizado, da teoria que os embasa, das diferenças metodológicas e da amostra estudada (que varia em função da idade, habilidade e nível educacional), os resultados têm apresentado amplas variações (Preckel et al., 2006). De uma forma geral, segundo Batey e Furnham (2006), as pesquisas sobre a relação entre criatividade e inteligência indicam que os dois construtos mostram-se modestamente relacionados (com correlações entre 0,20 e 0,40 ), de maneira a apontar que, a maior parte dos estudos, sugere que ambos não devem ser tratados como sinônimos, tal como foi relatado na presente pesquisa ao se encontrar diferentes fatores para os dois construtos.

Em relação ao segundo ponto destacado, a baixa correlação encontrada entre as medidas de criatividade verbal e figurativa (valores entre 0,04 e 0,15 na presente pesquisa), acompanha os resultados de outros estudos que, ao investigarem a performance nos dois tipos de criatividade, encontraram correlações modestas. Como exemplo pode-se citar o estudo de Cramond et al. (2005) cujo resultado apontou para um valor de correlação de 0,06, o estudo de Plucker (1999) de reanálise dos dados de Torrance $(\mathrm{r}=0,36)$ e a pesquisa de Clapham (2004), cujo valor também foi de 0,36, observado entre o escore total verbal e figural. Essas modestas correlações apontam para a constatação de que, segundo Silvia, Winterstien e Willse (2008), as tarefas do teste verbal e figural parecem avaliar diferentes traços por meio de diferentes métodos e não o mesmo traço por métodos diferentes.

Diante desse quadro, Zeng et al. (2011) argumenta acerca da predominância, errônea, de uma crença que os instrumentos podem prever o potencial criativo nos mais diversos domínios, contrariando a noção de criatividade enquanto domínio específico. Nesse mesmo sentido, Kim (2011) afirma que diversos autores chamam a atenção para o fato de que, ao tentar responder sobre a possibilidade de medir um construto tão complexo, a criatividade acaba, muitas vezes, sendo erroneamente tomada como um domínio geral, passível de ser avaliado por meio de um único instrumento. Na opinião dessa autora, tal ideia geraria, por si só, a crença de que se o indivíduo não é criativo em uma área, provavelmente não será em outras, de modo a desconsiderar a existência de evidências de que a criatividade seria composta por uma grande diversidade de domínios específicos, confirmada pelas baixas correlações encontradas entre diferentes medidas de criatividade (as quais se restringem, muitas vezes, a um único tipo de item ou tarefa), podendo-se citar ainda seus diferentes tipos (verbal ou figurativa, por exemplo), os quais podem capturar diferentes facetas da criatividade. Por essa razão, a autora salienta que os instrumentos deveriam ser usados somente no sentido de afirmar a criatividade, nunca de desconsiderá-la, visto que a performance criativa em uma tarefa poderia não predizer a performance criativa em outra.

Preocupação semelhante é apresentada por Silvia (2011) ao afirmar que a maior parte dos instrumentos usualmente é composta por diversos tipos de tarefas, cujas notas são posteriormente combinadas por meio de sua soma ou média simples, desconsiderando-se os diferentes processos criativos envolvidos em cada tipo de tarefa, de modo a afirmar sua equivalência e ignorar a possibilidade de que cada uma capture diferentes facetas da criatividade, assim como conhecimentos, estratégias e habilidades diferenciadas.

A partir dessas investigações, pode-se perceber a especificidade de cada tipo de criatividade, no presente estudo, a figurativa e a verbal, de modo a apontar particularidades que as diferenciam entre si. Representada através da produção de desenhos livres, a criatividade figural se apresenta como uma medida mais associada ao pensamento divergente (Wechsler, 2002) mobilizando uma série de características criativas que permitem a exploração da criatividade a ser manifestada através da descoberta de uma gama de respostas variadas diante de um mesmo estímulo/problema. 
Por sua vez, a produção metafórica, ao envolver um processo analógico, mobilizando habilidades mais relacionadas à associação entre palavras e significados estaria, conforme aponta Sternberg (1977), relacionada também ao raciocínio indutivo, permitindo a elaboração de associações consideradas criativas à medida que sejam capazes de demonstrar características comuns entre objetos, dentro de uma rede de significados que, a priori, se apresentariam semanticamente distantes, de modo a também exigir habilidades relacionadas à inteligência cristalizada.

Dadas as diferenças entre os dois tipos de criatividade estudadas, o que se pode afirmar é que a criatividade, manifestando-se de forma multifacetada, apresenta uma variedade de elementos que, de outra maneira, não se tornariam passíveis de observação e avaliação sem a utilização de instrumentos variados. Ressalta-se que no instrumento em estudo, dois tipos específicos de produção criativa foram avaliados, a figurativa e a verbal, destacando-se que outras expressões possam existir (musical, corporal etc).

Por fim, em relação ao terceiro ponto a ser discutido, as relações moderadas encontradas entre as medidas de criatividade verbal e inteligência, repetiram resultados apresentados por outras pesquisas, tais como a conduzida por Barros, Primi, Miguel, Almeida e Oliveira (2010) encontraram correlações de 0,31 entre a medida de criatividade metafórica e raciocínio abstrato e de 0,48 entre a medida de criatividade e raciocínio verbal, notadamente $\mathrm{RA}$ e $\mathrm{RV}$, mantendo com o primeiro uma correlação de magnitude menor quando comparada ao segundo, como a exemplo do que foi obtido nas análises realizadas neste estudo. Entretanto, apesar de valores de magnitude consideráveis observados nestas correlações, os autores citados, destacam que o raciocínio metafórico não deve se confundir com inteligência, antes deve ser entendido como um processo que além de demandar habilidades cognitivas e raciocínio analógico, também mobiliza habilidades relacionadas à produção criativa, ao empreendimento de novas e remotas associações, transposição de significados e construção de idéias novas e diversificadas. Também Silvia e Beaty (2012) constataram que sujeitos com inteligência fluida alta, apresentaram-se muito mais criativos na produção de metáforas, evidenciando um valor de correlação de 0,49 , destacando, no entanto, que a produção de metáforas trata-se de um processo que, além do pensamento divergente e características criativas, também está associado ao conhecimento cristalizado.

Desse modo, os resultados obtidos na presente pesquisa demonstram que a inteligência e criatividade verbal, apesar de manterem entre si relações e processos em comum, apresentam particularidades que justificam a avaliação de ambos os construtos, tal como foi proposto na bateria em estudo e cuja análise fatorial confirmou a separação de suas medidas.

\section{CONSIDERAÇÕES FINAIS}

Considerando-se o objetivo desta pesquisa, a busca por evidências de validade de construto da Bateria para Avaliação das Altas Habilidade, foi possível verificar a presença de três construtos avaliados pelo instrumento (criatividade verbal, inteligência e criatividade figural). A relevância desse tipo de investigação faz-se importante quando se trata de compreender a superdotação, devido às implicações que a definição do fenômeno determinam nas propostas e modelos de identificação. Isto porque, durante muito tempo, esse foi, erroneamente, tratado exclusivamente como sinônimo de um alto desempenho em testes de inteligência sendo que, dentro dos modelos mais atuais, esta teria, dentre seus componentes, a inteligência e criatividade, além de outros, tais como capacidade geral, liderança, talento artístico e aspectos motivacionais.

Ressalta-se que, apesar de se tratar aqui de um primeiro estudo psicométrico e, considerando-se ainda as limitações desta pesquisa, os resultados se fizeram importantes à medida que demonstraram a coerência da Bateria com o seu propósito inicial de avaliar construtos diferentes a partir dos seis subtestes que apresenta. Percebe-se a relevância desta pesquisa tanto pelos resultados favoráveis obtidos quanto às medidas propostas, quanto pela abertura de caminhos para investigação e confirmação de indicadores e condução de futuros estudos psicométricos com vista a busca por evidencias de outros tipos de evidências de validade, precisão e normatização deste instrumento para uso no Brasil.

Por fim, destaca-se que as altas habilidades/ superdotação é um fenômeno multidimensional e que sua identificação/avaliação implica também a mobilização de outros recursos que vão além do uso de instrumentos psicométricos, incluindo-se na bateria estudada, uma escala de avaliação a ser respondida pelo professor, cuja análise será foco de trabalho posterior. Entretanto, dentro do âmbito da avaliação psicológica, a grande contribuição do desta pesquisa refere-se, sobretudo, à contribuição que a mesma poderá trazer à área da avaliação psicológica no sentido de amenizar uma situação atestada no Brasil que aponta para a falta de testes psicológicos validados e normatizados para identificação das reais potencialidades das crianças com altas habilidades/superdotação. 


\section{REFERÊNCIAS}

Aguirre, K., \& Conners, F. (2010). Creativity and intelligence in preschoolers: preliminary findings. The University of Alabama McNair Journal. Recuperado em 15 de março, 2013, de http:// graduate.ua.edu/mcnair/journals/2010/Aguirre.pdf

Albert, R.S., \& Runco, M. A. (1999). A history of research on creativity. In R.J. Sternberg. Handbook of creativity (pp. 16-31). Cambridge: Cambridge University Press.

Almeida, M. A., \& Capellini, V. L. M. F. (2005). Alunos talentosos: possíveis superdotados não identificados. Educação, 1(55), 45-64.

Barros, D. P., Primi, R., Miguel, F. K., Almeida, L., \& Oliveira, E. P. (2010). Metaphor creation: A measure of creativity or intelligence? European Journal of Education and Psychology, 3(1), 103-115.

Batey, M., \& Furnham, A. (2006). Creativity, intelligence and personality: a critical review of the scattered literature. Genetic, Social and General Psychology Monographs, 132(4), 355-429.

Bracken, B. A., \& Brown, E. F. (2006). Behavioral identification and assessment of gifted and talented students. Journal of Psychological Assessment, 24(2), 112-122.

Clapham, M. M. (2004). The convergent validity of the Torrance Tests of Creative Thinking and Creative Interest Inventories. Educational and Psychological Measurement, 64, 828-841.

Colom, R. (2008). Nos limites da inteligência: é o ingrediente do êxito na vida? (C. E. Flores-Mendoza \& F.M. Franco, trad.). São Paulo: Vetor.

Cramond, B., Matthews-Morgan, J., Bandalos, D., \& Zuo, L. (2005). A report on the 40-year follow-up of the Torrance Tests of Creative Thinking. Gifted Child Quarterly, 49, 283-356.

Elisondo, R. C., \& Donolo, D. S. (2010). ¿Creatividad o inteligencia? That is not the question. Anales de Psicología, 26(2), 220-225.

Gardner, H (2001). Inteligência: um conceito reformulado. Rio de Janeiro: Objetiva.

Getzels, J. W., \& Jackson, P. W. (1962). Creativity and intelligence: explorations with gifted students. Wiley, NY.

Gonçalves, F. C., \& Fleith, D. F. (2011). Estudo comparativo entre alunos superdotados e não superdotados em relação à inteligência e criatividade. Psico, 42(2), 263-268.

Gridley, B. E., \& Treloar, J. H. (1984). The validity of the scales for rating the behavioral characteristics of superior students for the identification of gifted students. Journal of Psychoeducational Assessment, 2(1), 65-71.

Hattie, J., \& Rogers, H.J. (1986). Factor Models for Assessing the Relation Between Creativity and Intelligence. Journal of Educational Psychology, 78(6), 482-485.

Holling, H., \& Kuhn, J. T. (2008). Does intellectual giftedness affect the factor structure of divergent thinking? Evidence from a MG-MACS analysis. Psychology Science Quarterly, 50(2), 283-294.

Kim, K. H. (2005). Can only intelligent people be creative? A meta-analysis. The Journal of Secondary Gifted Education, 16, 57-66.

Kim, K. H. (2011). The APA 2009 division 10 debate: are the Torrance Tests of Creative Thinking still relevant in the $21 \mathrm{st}$ century? Psychology of Aesthetics, Creativity, and the Arts. [Online publication].

Li, H., Lee, D., Pfeiffer, S. I., Kamata, A., Kumtepe, A. T., \& Rosado, J. (2009). Measurement invariance of the Gifted Rating Scales - school form across five cultural groups. School Psychology Quaterly, 24(3), 186-198.
MEC (2010). Políticas Públicas para as Altas Habilidades/ Superdotação. Recuperado em 01 de março 2013, de http:// www.senado.gov.br/web/comissoes/CE/AP/AP20080626_ superdotados_Cl\%C3\%AludiaGriboski.pdf

Muthén, L. K., \& Muthén, B. O. (2010). Mplus User's Guide ( $6^{\mathrm{a}}$ ed.). Los Angeles, CA: Muthén \& Muthén.

Nakano, T. C., \& Brito, M. E. (no prelo). Avaliação da criatividade a partir do controle do nível de inteligência em uma amostra de crianças. Temas em Psicologia.

Nakano, T. C., \& Primi, R. (2012). Bateria de avaliação das altas habilidades. Não publicado.

Pereira, M. (2001). Inteligência e criatividade: duas trajectórias alternativas para as crianças sobredotadas? Psicologia: Teoria, Investigação e Prática, 6(1), 171-188.

Pfeiffer, S. I., \& Blei, S. (2008). Gifted identification beyond the IQ test: rating scales and other assessment procedures. In S. I. Pfeiffer (Org.). Handbook of giftedness in children: psychoeducational theory, research and best practices (pp. 177-198). New York: Springer.

Plucker, J. A. (1999). Is the proof in the pudding? Reanalyses of Torrance's (1958 to present) longitudinal data. Creativity Research Journal, 12, 103-114.

Preckel, F., Holling, H., \& Wiese, M. (2006). Relationship of intelligence and creativity in gifted and non-gifted students: An investigation of threshold theory. Personality and Individual Differences, 40, 159-170.

Primi, R., Miguel, F., Couto, G., \& Muniz, M. (2007). Precisão de avaliadores na avaliação da criatividade por meio da produção de metáforas. Psico-USF, 12(2), 197-210.

Renzulli, J. S. (1978). What makes giftedness? reexamining a definition. Phi Delta Kappan, 60(5), 180-184.

Renzulli, J. S. (2004). O que é esta coisa chamada superdotação, e como a desenvolvemos? Uma retrospectiva de vinte e cinco anos. Revista Educação, 52(1), 75-131.

Rindermann, H., \& Neubauer, A. C. (2004). Processing speed, intelligence, creativity, and school performance: Testing of causal hypotheses using structural equation models. Intelligence, 32, 573-589.

Roazzi, A., Dias. M. G. B. B., Athias, R., Brandão, M. C., Souza, B. C., \& O'Brien, D. (2007). Inteligência, processos mentais e contexto cultural. In A. A. Candeias \& L. S. Almeida (Orgs.). Inteligência humana: investigação e aplicações (pp. 19-50). Évora: Quarteto.

Silvia, P. J. (2011). Subjective scoring of divergent thinking: examining the reliability of unusual uses, instances and consequences tasks. Thinking Skills and Creativity, 6, 24-30.

Silvia, P. J., Winterstein, B. P., \& Willse, J. T. (2008). Rejoinder: The Madness to Our Method: Some Thoughts on Divergent Thinking. Psychology of Aesthetics, Creativity, and the Arts, 2(2), 109-114.

Silvia, P. J., \& Beaty, R. E. (2012). Making creative metaphors: the importance of fluid intelligence for creative thought. Intelligence, 40(4), 343-354.

Sternberg, R. J. (1977). A Component Process in Analogical Reasoning Psychological Review, 84(4), 353-378.

Sternberg, R. J. (1984). What should intelligence tests test? Implications of a triarchic theory of intelligence for intelligence testing. Educational Researcher, 13, 5-15.

Sternberg, R. J. (2005). The theory of sucessfull intelligence. Interamerican Journal of Psychology, 39(2), 189-202.

Sternberg, R. J., \& O'Hara, L. A. (2000). Intelligence and creativity. In R. J. Sternberg (Ed.). Handbook of intelligence (pp. 611-630). New York, NY: Cambridge University Press. 
Wechsler, S. M. (2002). Avaliação da criatividade por figuras e palavras. Teste de Torrance, versão brasileira. LAMP/PUC. Campinas: Impressão Digital do Brasil Gráfica e Editora.

Wechsler, S. M., Nakano, T. C., Nunes, M. F. O., \& Minervino, C. A. M. (2010). Avaliação cognitiva de crianças e jovens: aspectos multidimensionais. In C. S. Hutz (Org.). Avanços em avaliação psicológica e neuropsicológica de crianças e adolescentes (pp. 31-68). São Paulo: Casa do Psicólogo.

Wechsler, S. M., Nunes, M. F. O., Schelini, P. W., Ferreira, A. A., \& Pereira, D. A. P. (2010). Criatividade e inteligência: analisando semelhanças e discrepâncias no desenvolvimento. Estudos de Psicologia, 15(3), 243-250.

Zeng, L., Proctor, R. W., \& Salvendy, G. (2011). Can traditional divergent thinking tests be trusted in measuring and predicting real-world creativity? Creativity Research Journal, 23(1), 24-37.
Agradecimento:

Os autores agradecem ao CNPq e a Capes pelo apoio recebido e à Priscila Zaia pelo auxílio na aplicação e correção dos instrumentos.

Autores:

Walquiria de Jesus Ribeiro - Mestranda em Psicologia pela PUC-Campinas, Bolsista Capes II.

Tatiana de Cássia Nakano - Docente do Curso de Pós-Graduação em Psicologia da PUC-Campinas.

Ricardo Primi - Docente do Curso de Pós-Graduação em Psicologia da Universidade São Francisco.

Endereço para correspondência:

Walquiria de Jesus Ribeiro

Rua Minas Gerais, 875, Chácara Brasil - Turú

CEP: 65065-770 São Luis, MA, Brasil

Tel.: (19)8177-3750 - (98)3242-7561

E-mail: walquiriaribeiro@hotmail.com

Recebido em: 29.04.2013.

Aceito em: 01.10.2013. 